\title{
Colorectal endoscopic submucosal dissection using the pocket-creation method with single clip traction: a feasibility study $\boldsymbol{\nabla}$
}

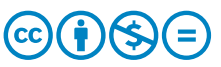

\author{
Authors \\ Keijiro Sunada', Alan Kawarai Lefor ${ }^{2}$, Hironori Yamamoto ${ }^{1}$ \\ Institutions \\ 1 Department of Medicine, Division of Gastroenterology, \\ Jichi Medical University, Shimotsuke, Japan \\ 2 Department of Surgery, Jichi Medical University, \\ Shimotsuke, Japan
}

Tatsuma Nomura ${ }^{1}$, Yoshikazu Hayashi' ${ }^{1}$ Takaaki Morikawa', Masahiro Okada ${ }^{1}$, Hisashi Fukuda', Takahito Takezawa ${ }^{1}$,

submitted 13.9.2020

accepted after revision 9.12 .2020

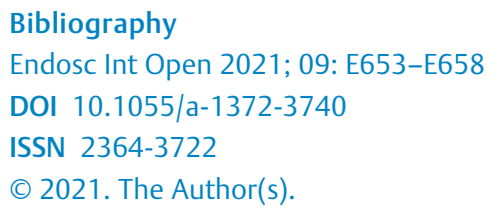
Commons Attribution-NonDerivative-NonCommercial License, permitting copying and reproduction so long as the original work is given appropriate credit. Contents may not be used for commercial purposes, or adapted, remixed, transformed or built upon. (https://creativecommons.org/licenses/by-nc-nd/4.0/)

Georg Thieme Verlag KG, Rüdigerstraße 14,

70469 Stuttgart, Germany

Corresponding author

Hironori Yamamoto, MD, PhD, Department of Medicine, Division of Gastroenterology, Jichi Medical University, Shimotsuke, 3311-1 Yakushiji, Shimotsuke, Tochigi, 3290498, Japan

Fax: +81-285-40-6598

ireef@jichi.ac.jp

\section{ABSTRACT}

Background and study aims The pocket-creation method (PCM) facilitates dissection of the central part of a tumor. We previously developed the PCM with clip traction (PCM-CT) to facilitate opening the mucosal pocket, which otherwise could become cumbersome. In the present study, we aimed to examine the feasibility of PCM-CT for colorectal endoscopic submucosal dissection (ESD).

Patients and methods PCM-CT was performed on 30 patients with early colorectal tumors from October 2019 to April 2020. PCM-CT allows efficient opening of the mucosal pocket by using the PCM to dissect the center of the lesion and then apply traction with a single clip after making a circumferential mucosal incision.

Results The median specimen major axis length, ESD time, ESD speed, and en bloc resection rate were $48 \mathrm{~mm}, 84 \mathrm{~min}-$ utes, $20 \mathrm{~mm}^{2} / \mathrm{min}$, and $100 \%$ (30/30), respectively. The success rates for the traction clip and median single-cliptraction time were $100 \%$ (30/30) and 1.5 minutes, respectively.

Conclusions Colorectal ESD using PCM-CT is a simple and promising method.

\section{Introduction}

The visibility of the submucosa is a crucial step for the efficacy and safety of endoscopic mucosal dissection (ESD). Therefore, in recent years, many traction methods have been devised to facilitate ESD [1,2]. However, the treatment of lateral spreading tumors, non-granular type (LST-NG) lesions with extensive fibrosis, and large Paris type 0 -Is lesions is still difficult [3]. We previously devised the pocket-creation method (PCM) that creates a submucosal pocket, maintains the visibility of the submucosal layer in a hood with a tapered tip, and enables en bloc removal of tumors with extensive fibrosis $[4,5]$. In the pocket, the tip of the endoscope is parallel to the muscle layer, which allows appropriate visualization of the submucosa. Traction provided by a tapered hood can easily provide visibility of the submucosa above the muscularis layer. The tip of the scope enveloped in the submucosal pocket can maintain its stability while being synchronized with the patient's cardiac and respiratory movements. We usually used natural traction such as gravity and/or intentionally leaving a portion of the submucosal tissue when opening the pocket when using the PCM. However, some endoscopists have struggled to remove the remaining 

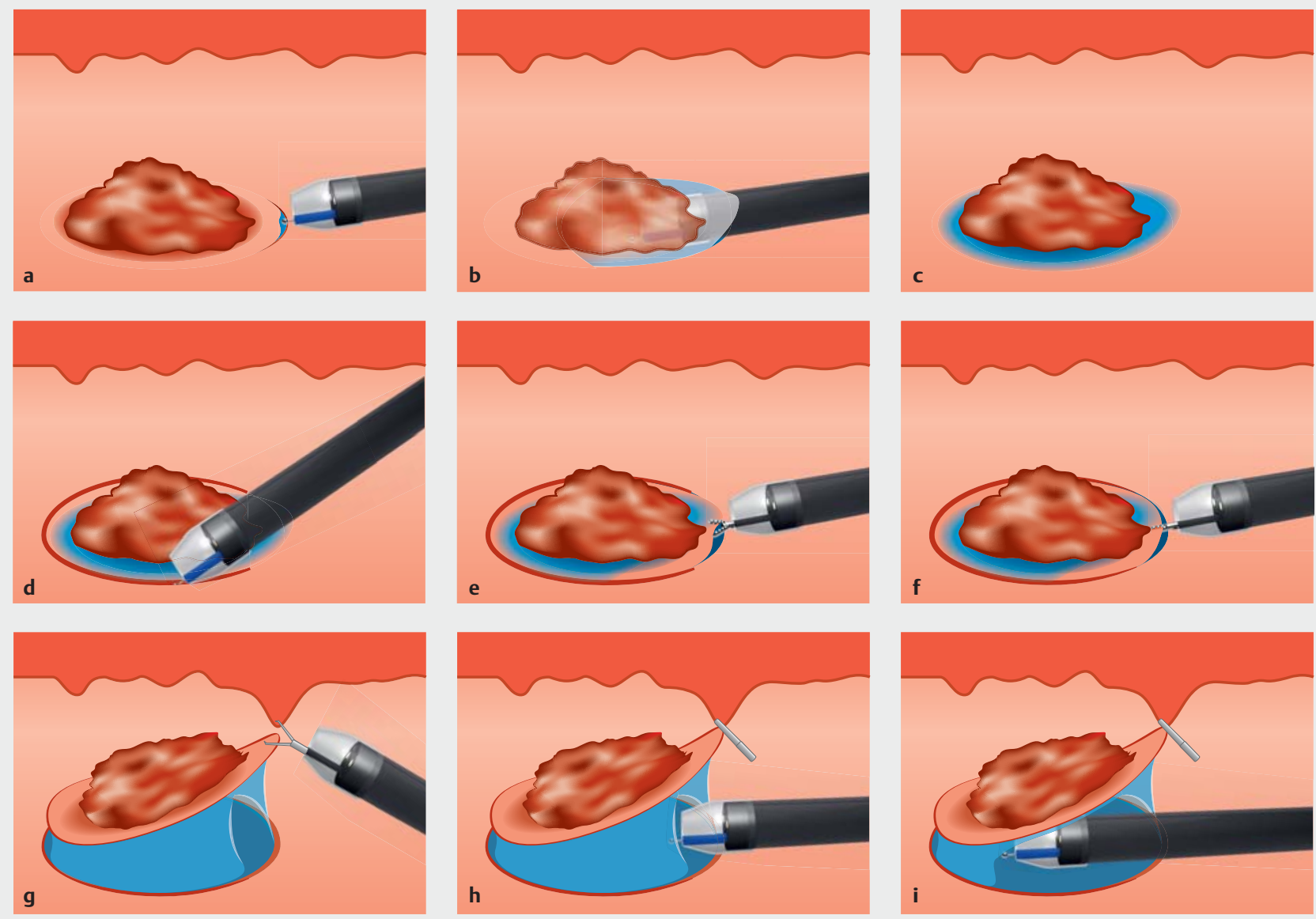

- Fig. 1 Schema of colorectal endoscopic submucosal dissection using the pocket-creation method with single clip traction (PCM-CT) a First mucosal incision. $\mathbf{b}$ Submucosal pocket creation. $\mathbf{c}$ Dissection under the lesion using PCM. $\mathbf{d}$ Circumferential mucosal incision around the lesion. e Normal mucosa on the distal edge of the partially resected lesion is grasped with a reopenable clip, without deploying it. $\mathbf{f}, \mathbf{g}, \mathbf{h}$ The entrapped mucosa is pulled towards the opposite colonic wall and the clip is slowly opened to further capture the opposing colonic mucosa. Capture of both the lesion and opposing colonic mucosa is visually confirmed and then the clip is deployed. I PCM-CT stretches the submucosa, exposing the ideal submucosal dissection line, allowing it to be safely dissected.

submucosa around the lesion if the submucosa has already been dissected and gravity traction is not effective or when the endoscopist is less experienced. Therefore, we devised the PCM with single clip traction (PCM-CT), as a novel method of adding traction with a reopenable clip while opening the pocket [6]. We present the feasibility of PCM-CT for colorectal ESD.

\section{Patients and methods}

\section{Patients}

We retrospectively analyzed the first 30 consecutive patients who underwent PCM-CT among a total of 80 cases of ESD for early colorectal tumors at Jichi Medical University Hospital between October 2019 and April 2020. All patients provided their consent before undergoing colorectal ESD using PCM-CT, and this study was approved by the ethics committee of the Jichi Medical University, Tochigi, Japan.

\section{ESD device}

A therapeutic colonoscope with a waterjet channel (EC-580RD/ M; Fujifilm, Tokyo, Japan), a water irrigator (JW-2; Fujifilm) and a small-caliber-tip transparent (ST) hood (DH-33GR [latest ST hood]; Fujifilm) were used. When a standard colonoscope could display the lesion but had inadequate maneuverability during the preoperative colonoscopy, a balloon-assisted endoscope (EI-580BT with TS-13101; Fujifilm) was occasionally used as previously reported [7]. For mucosal incision and submucosal dissection, Dualknife (KD-650Q; Olympus, Tokyo, Japan) or Flushknife (DK2618JB-15; Fujifilm) was used. For submucosal injection, $0.4 \%$ sodium hyaluronate (MucoUp; Boston scientific Co, Tokyo, Japan) was used.

\section{PCM-CT procedure}

A submucosal pocket was first created by dissecting the submucosa beneath the lesion as a part of conventional PCM ( $\vee$ Fig. 1, $>$ Fig. 2, $\vee$ Video 1). The edge of the ST hood stretched the submucosa and held the plane of the muscularis pro- 

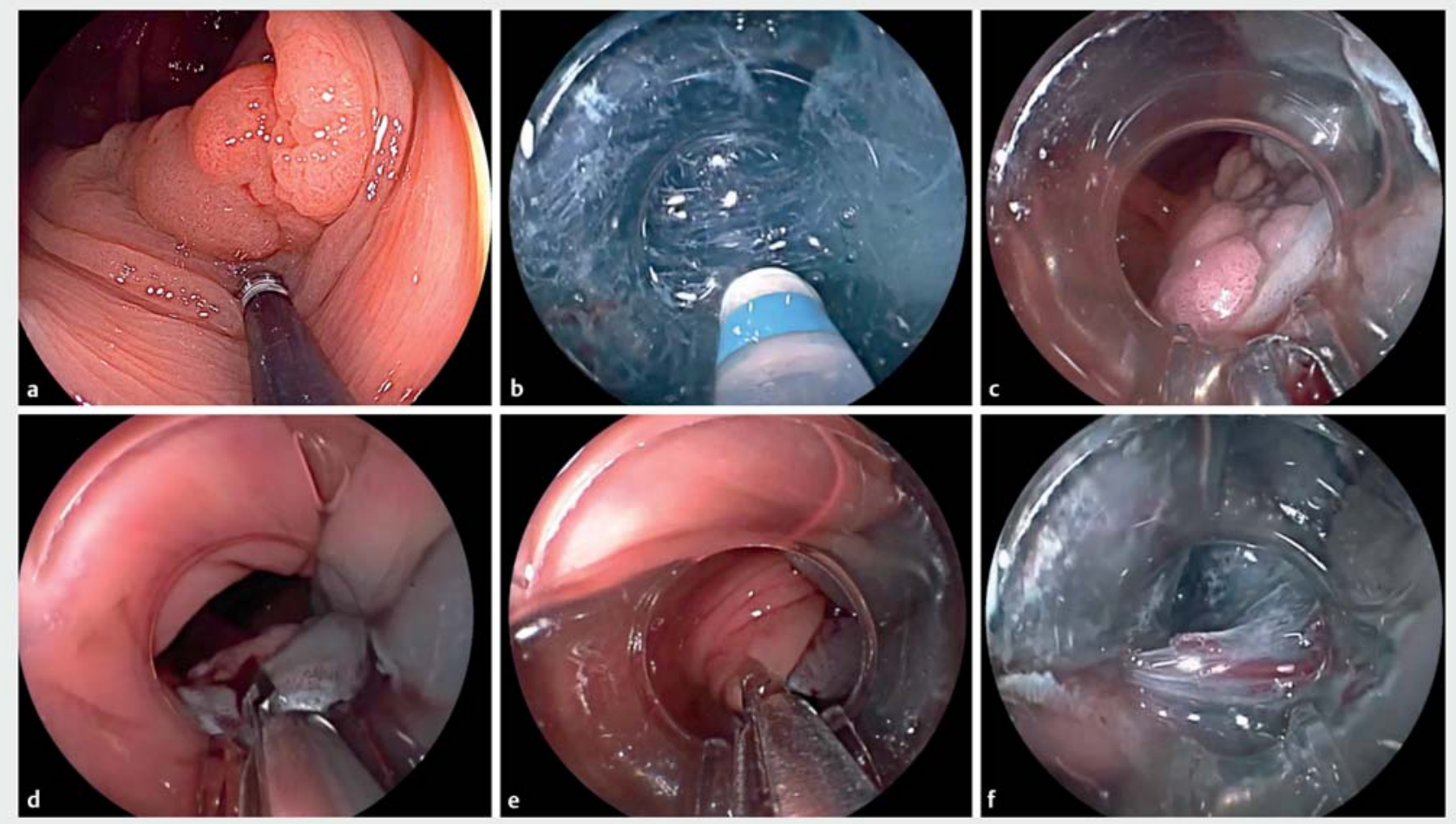

- Fig. 2 Representative images of original colorectal endoscopic submucosal dissection (ESD) using the pocket-creation method with single clip traction (PCM-CT). a A 30-mm, Paris Ila, nongranular, JNET 2B, laterally spreading lesion in the ascending colon. b Colonic ESD being performed using PCM. c A circumferential incision being made in the normal mucosa around the lesion after the submucosal pocket has been created. $\mathbf{d}$ Normal mucosa on the distal edge of the lesion being captured with a reopenable clip. e The clip, with the captured mucosa from the edge of the lesion, being pulled towards the opposite colonic wall and reopened to entrap the opposing normal colonic mucosa as well. f Identification of the safest submucosal dissection line is facilitated by PCM with clip traction.

pria, which also allowed a precise submucosal dissection just above the muscularis propria. After creating the submucosal pocket under the lesion, a circumferential mucosal incision was made $7 \mathrm{~mm}$ outside the lesion, measured by the $7-\mathrm{mm}$ diameter of the ST hood. Subsequently, single-clip traction (the PCM-CT) was performed with a 16-mm-wide reopenable clip (SureClip; Micro-Tech Co. Ltd., Nanjing, China). The distal edge of the partially resected specimen was grasped by the clip without being deployed. The entrapped specimen was pulled towards the opposite colonic wall. When deflating the colon, its lumen will collapse, which facilitates connecting the abutting colonic walls with a SureClip. The clip was carefully opened to capture additional opposing colonic mucosa. Capture of both the resected specimen and the opposite colonic mucosa was visually confirmed. Then the clip was deployed to connect them. The clip traction stretched the remaining submucosa around the pocket, which exposed the ideal dissection line on the submucosa and allowed a safe submucosal dissection with minimal thermal energy from the electrocautery and no damage to either the resected specimen or the muscularis propria. When controlling the amount of gas in the intestinal tract lumen by insufflation or deflation, PCM-CT can adjust its traction appropriately without using a spring [8] or rubber band [9]. Even in the deflated situation, advancing the SThood tip can apply traction to the submucosa in front of it be- cause the distal edge of the dissected portion is fixed to the opposite wall. Finally, the clip that is still holding the completely resected specimen was easily removed by pulling back on its arms using a regular or a toothed grasping forceps. The ESD specimen is not damaged during removal from the opposite wall because the ESD specimen has already been freed from any wall and moves with the clip. Therefore, the specimen is retrieved with the attached clip. The clip is removed before pin-

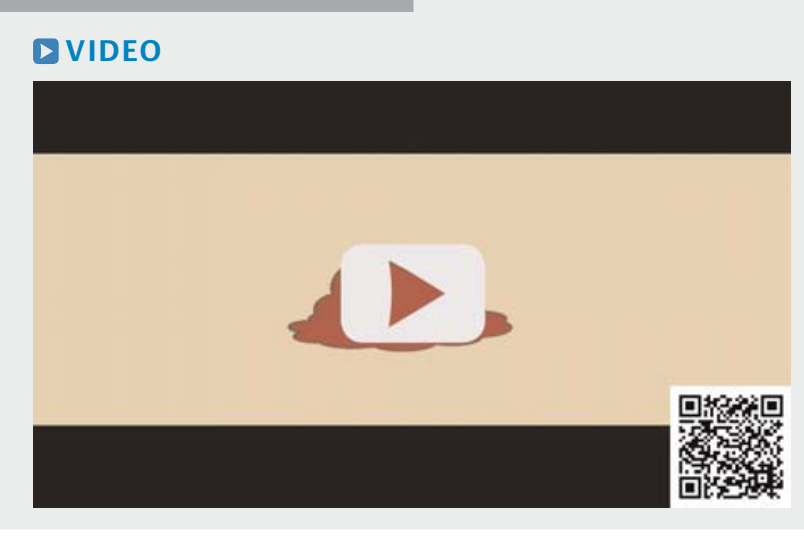

Video 1 A schema and an example of colorectal ESD using PCM-CT 
ning the specimen because the clip which grips the distal margin may make pathological evaluation of the margin incorrect. The clip is easily removed from the specimen without damaging the specimen by folding one of the two teeth of the clip with forceps. All ESDs were performed by 10 endoscopists. Six of them were expert endoscopists. Four trainees with experience of less than 20 ESDs performed the ESDs under direct supervision of an expert [10].

\section{Evaluation of colorectal ESD}

During the ESD procedures, the presence and extent of the submucosal fibrosis were assessed according to a previously reported system [11]. Pocket-creation time was defined as the time from the first mucosal incision to the beginning of the circumferential mucosal incision. The circumferential-mucosal-incision time was defined as the period from the start of full circumferential mucosal incision to the appearance of the reopenable clip on the display screen. The single-clip-traction time was defined as the time from appearance of the reopenable clip on the display screen until it is deployed. The pocket-opening time was defined as the time from the total incision to the end of the resection of the lesion. The dissection speed $\left(\mathrm{mm}^{2} /\right.$ min) was calculated by using the area of the resected specimen $\left(\mathrm{mm}^{2}\right)$ /dissection time (minutes). En bloc resection was defined as a tumor removed as a single piece. $\mathrm{R} 0$ resection was defined as an en bloc resection with pathologically negative resection margins (vertical and lateral) [12]. PCM-CT was considered successful when the mucosa on the lesion side and the normal mucosa on the contralateral colorectal wall were firmly connected after deploying the clip. Dropping of PCM-CT was defined as dropping the traction clip which provided appropriate traction when it was deployed when opening the pocket.

\section{Results}

- Table 1 shows the characteristics of lesions in patients who underwent PCM-CT. The median lengths along the major axes of the resected specimens and major axes of the tumors in the resected specimens were $48 \mathrm{~mm}$ and $31 \mathrm{~mm}$, respectively. Of 30 lesions, balloon-assisted ESD (BAESD) was performed for six of the lesions located in the ascending and transverse colon. The en bloc resection rate was $100 \%$ (30/30), the R0 resection rate was $87 \%(26 / 30)$, and the negative vertical margin rate was $100 \%(30 / 30)$.

- Table 2 shows the outcomes of ESD using PCM-CT. The dissection speed was $20 \mathrm{~mm}^{2} / \mathrm{min}$. The success rate for placing the traction clip was $100 \%$. The median procedure time for placement of the clip was 1.5 minutes. Dropping the clip while opening the pocket was observed in two of 30 procedures. However, the procedures were repeated with deployment of another clip in both cases without any adverse events.

\section{Discussion}

In 30 consecutive cases of ESD using PCM-CT, the median length along the major axis of the resected-specimen was 48 $\mathrm{mm}$, the median procedure time was 84 minutes, and the me-
- Table 1 Characteristics of patients and lesions treated using the pocket-creation method with single clip traction.

\begin{tabular}{|l|l|}
\hline Characteristic & Value \\
\hline Age (median), years & 69 \\
\hline Gender & \\
\hline Male/female & $17 / 13$ \\
\hline Lesion location & \\
\hline C/A/T/D/S/R & $5 / 8 / 9 / 2 / 4 / 2$ \\
\hline Lesion classification & \\
\hline LST-G/ LST-NG/ non LST & $12 / 10 / 8$ \\
\hline Fibrosis & \\
\hline F0/F1/F2 & $14 / 13 / 3$ \\
\hline Tumor and resected specimen size & $31(10-125)$ \\
\hline Tumor major axis (mm), median (range) & $26(10-80)$ \\
\hline Tumor minor axis (mm), median (range) & $48(25-150)$ \\
\hline Specimen major (mm), median (range) & $40(25-105)$ \\
\hline Specimen minor (mm), median (range) & 13 \\
\hline Histopathology & 4 \\
\hline Adenoma & \\
\hline Intramucosal carcinoma & \\
\hline Slightly invasive (<1000 $\mu$ m) submucosal carcinoma & 4 \\
\hline Deeply invasive ( $\geq 1000 \mu m)$ submucosal carcinoma & 2 \\
\hline $\begin{array}{l}\text { C, cecum; A, ascending; T, transverse; D, descending; S, sigmoid; Upper R, } \\
\text { erally spreading tumor, non-granular type }\end{array}$ & \\
\hline
\end{tabular}

- Table 2 Outcomes of endoscopic submucosal dissection using the pocket-creation method with single clip traction.

\begin{tabular}{|l|l|}
\hline Procedure time & $84(30-414)$ \\
\hline Dissection time & $50(25-173)$ \\
\hline \begin{tabular}{l} 
Pocket-creation time (min), median (range) \\
\hline $\begin{array}{l}\text { Circumferential-mucosal-incision time (min), } \\
\text { median (range) }\end{array}$
\end{tabular} & $12(3-140)$ \\
\hline Pocket-opening time (min), median (range) & $33(11-244)$ \\
\hline Procedure speed & $20(8-39)$ \\
\hline Dissection speed (mm²/min), median (range) & $100 \%(30 / 30)$ \\
\hline PCM-CT procedure & $1.5(1-6)$ \\
\hline Success rate of the traction clip, $\mathrm{n}$ & $7 \%(2 / 30)$ \\
\hline Single-clip-traction time (min), median (range) & \\
\hline Clip-dropping rate, $\mathrm{n}$ & \\
\hline PCM-CT, pocket-creation method with clip traction. & \\
\hline
\end{tabular}


dian dissection speed was $20 \mathrm{~mm}^{2} / \mathrm{min}$. In a multicenter randomized controlled trial (RCT) of the reports, Yamashina et al. reported a specimens median major axis length of $35 \mathrm{~mm}$, procedure time of 51 minutes, and dissection speed of $16 \mathrm{~mm}^{2} /$ min using PCM [13]. PCM can reduce respiratory movements, allow the submucosal layer to be approached tangentially to the muscular layer by facilitating traction of the submucosal layer with an ST hood, maintain the bulge of the submucosal layer with pathologically negative resection margins, and achieve a high rate of en bloc excision [14]. However, it is difficult to continue to make use of these advantages when opening the pocket. PCM-CT is a promising new approach that maintains a high en bloc resection rate and efficient dissection because it allows the submucosal layer to be visible and dissected even when opening the pocket.

In the present study, the median single-clip-traction time was 1.5 minutes, and the success rate for placement of the clip was $100 \%$. Because we used a reopenable clip, the first-time PCM-CT success rate was $100 \%$ (30/30). The reason for this success is that we could deploy the clip after repeatedly confirming appropriate clip-deployment where the clip provides significant traction to the lesion in the endoscopic view. However, there were two cases in which the traction clips dropped when opening the pocket. In both of these cases, too little contralateral normal mucosa was grasped, and the clip dropped during the procedure. However, another reopenable clip was placed to regain traction in both cases. As the median single-clip-traction time of the 30 ESDs was just 1.5 minutes, the single-clip traction procedure may be easier and simpler than using other traction devices. Even if the first traction clip drops off unexpectedly, we only need to place another reopenable clip to regain traction because it does not require complicated preparation and can be performed without withdrawing the endoscope. Single-clip traction can be added to an ESD procedure on demand, in a different manner from some dedicated ESD devices such as "tissue retractor system [1]" and "double balloon endoluminal platform [15]." Even if we were not going to use single-clip traction when beginning ESD, we can add singleclip traction during the ESD procedure on demand.

Yamasaki et al. reported on traction-assisted colonic endoscopic submucosal dissection using clip and line with a success rate of $95 \%$ (40/42), and PCM-CT has shown a similar success rate [16]. Meanwhile, even if the first traction clip cannot keep enough traction in $\mathrm{PCM}-\mathrm{CT}$, we can place more clips to have additional traction. S-O Clip traction and magnetic anchor-guided ESD dissection (MAG-ESD) have been also reported as useful methods that can add traction to the contralateral side of the lesion. However, they require a longer procedure time than PCM-CT (4.4 min and 8 min, respectively) $[17,18]$.

Damage to the ESD specimen or bleeding from the oppositewall mucosa after removing the clip may be a concern when performing PCM-CT. However, these were not major problems even if they occurred. The distal normal mucosa is usually larger than surrounding normal mucosa in the specimen resected using the PCM because the initial mucosal incision is always made in 1.5 to $2 \mathrm{~cm}$ from the distal edge of the tumor in PCM. Therefore, it was easy to grasp the distal normal mucosa with- out involving the tumor. Even if the tumor was grasped by the clip temporarily, we could also regrasp it so as not to pierce the tumor. Although slight bleeding occasionally occurred from the torn mucosa on the opposite wall after removing the clip, hemostasis was quickly achieved as after a typical biopsy. PCMCT may not be better for rectal lesions near the anal canal compared with other colorectal lesions because there is little space for clip placement in the anal canal. When a rectal lesion is near the anal canal, the "pocket-creation method with pulley clip elastic line traction” we reported recently will be preferred [19].

\section{Conclusions}

Colorectal ESD using PCM-CT is a promising and novel method. This is a relatively small feasibility study and cannot be compared to existing PCM data. Even PCM-CT demanded that we train for its reliable performance. In addition, selection bias cannot be eliminated if we compare PCM-CT and PCM in this cohort because either PCM-CT or PCM was chosen by each endoscopist according to their preferences and experiences. Therefore, we plan to conduct a multicenter RCT for colorectal ESD using the PCM-CT and conventional PCM to account for these factors.

\section{Competing interests}

Dr. Yamamoto has a patent for the ST hood produced by the Fujifilm Corporation. The hood was used for ESD and shown in this video. He also has a consultant relationship with the Fujifilm Corporation and has received honoraria, grants, and royalties from the company.

\section{References}

[1] Jawaid S, Yang D, Draganov PV. Tissue retractor system-assisted endoscopic submucosal dissection of a large rectal tumor with significant fibrosis from direct tattooing. VideoGIE 2019; 4: 84-86

[2] Sakamoto N, Osada T, Shibuya T et al. The facilitation of a new traction device ( $\mathrm{S}-\mathrm{O}$ clip) assisting endoscopic submucosal dissection for superficial colorectal neoplasms. Endoscopy 2008; 40: E94-E95

[3] Moss A, Williams S], Hourigan LF et al. Long-term adenoma recurrence following wide-field endoscopic mucosal resection (WF-EMR) for advanced colonic mucosal neoplasia is infrequent: results and risk factors in 1000 cases from the Australian Colonic EMR (ACE) study. Gut 2015; 64: 57-65

[4] Hayashi Y, Miura Y, Yamamoto H. Pocket-creation method for the safe, reliable, and efficient endoscopic submucosal dissection of colorectal lateral spreading tumors. Dig Endosc 2015; 27: 534-535

[5] Hayashi Y, Sunada K, Takahashi H et al. Pocket-creation method of endoscopic submucosal dissection to achieve en bloc resection of giant colorectal subpedunculated neoplastic lesions. Endoscopy 2014; 46: E421-E422

[6] Nomura T, Hayashi Y, Lee RF et al. Pocket-creation method using a new technique of single-clip traction for colorectal endoscopic submucosal dissection. Endoscopy 2020; 52: E208-E210

[7] Yamashina T, Hayashi Y, Sakamoto $\mathrm{H}$ et al. Balloon-assisted endoscopy facilitates endoscopic submucosal dissection of difficult superficial proximal colon tumors. Endoscopy 2018; 50: 800-808 
[8] Okamoto Y, Oka S, Tanaka S et al. Clinical usefulness of the S-O clip during colorectal endoscopic submucosal dissection in difficult-toaccess submucosal layer. Endosc Int Open 2020; 8: E437-E449

[9] Jacques ], Charissoux A, Legros $R$ et al. Double clip counter traction using a rubber band is a useful and adaptive tool for colonic endoscopic submucosal dissection. Endoscopy 2018; 50: 179-181

[10] Nomura T, Miura Y, Yamamoto H. Novel approach to training novices in endoscopic submucosal dissection: The Master-Apprentice Technique. Dig Endosc 2020; 32: e30-e31

[11] Matsumoto A, Tanaka S, Oba S et al. Outcome of endoscopic submucosal dissection for colorectal tumors accompanied by fibrosis. Scand J Gastroenterol 2010; 45: 1329-1337

[12] Yamashina T, Hayashi Y, Fukuda $\mathrm{H}$ et al. The pocket-creation method may facilitate endoscopic submucosal dissection of large colorectal sessile tumors. Endosc Int Open 2020; 8: E1021

[13] Yamashina T, Nemoto D, Hayashi Y et al. Prospective randomized trial comparing the pocket-creation method and conventional method of colorectal endoscopic submucosal dissection. Gastrointest Endosc 2020: doi:10.1016/j.gie.2020.02.034
[14] Sakamoto H, Hayashi Y, Miura Y et al. Pocket-creation method facilitates endoscopic submucosal dissection of colorectal laterally spreading tumors, non-granular type. Endosc Int Open 2017; 5: E123-E129

[15] Ismail MS, Bahdi F, Mercado MO et al. ESD with double-balloon endoluminal intervention platform versus standard ESD for management of colon polyps. Endosc Int Open 2020; 8: E1273-E1279

[16] Yamasaki Y, Takeuchi $\mathrm{Y}$, Uedo $\mathrm{N}$ et al. Efficacy of traction-assisted colorectal endoscopic submucosal dissection using a clip-and-thread technique: a prospective randomized study. Dig Endosc 2018; 30: 467-476

[17] Hashimoto R, Hirasawa D, Iwaki T et al. Usefulness of the S-O clip for gastric endoscopic submucosal dissection (with video). Surg Endosc 2018; 32: 908-914

[18] Matsuzaki I, Hattori M, Yamauchi $\mathrm{H}$ et al. Magnetic anchor-guided endoscopic submucosal dissection for colorectal tumors (with video). Surg Endosc 2020; 34: 1012-1018

[19] Nomura T, Hayashi Y, Yamamoto H. Pocket-creation method with pulley clip elastic line traction for large rectal tumors. Dig Endosc 2020: doi:10.1111/den.13749 